\title{
Circulating plasma proteins and new-onset diabetes in a population-based study: proteomic and genomic insights from the STANISLAS cohort
}

\author{
João Pedro Ferreira®1, Zohra Lamiral' ${ }^{1}$, Constance Xhaard'1 Kévin Duarte' ${ }^{1}$, Emmanuel Bresso², \\ Marie-Dominique Devignes², Edith Le Floch ${ }^{3}$, Claire Dandine Roulland ${ }^{3}$, Jean-François Deleuze ${ }^{3}$, Sandra Wagner', \\ Bruno Guerci ${ }^{4}$, Nicolas Girerd ${ }^{1}$, Faiez Zannad ${ }^{1}$, Jean-Marc Boivin' ${ }^{1}$ and Patrick Rossignol $^{\mathbf{1}}$ \\ ${ }^{1}$ Université de Lorraine, INSERM, Centre d'Investigations Cliniques Plurithématique 1433, INSERM 1116, CHRU de \\ Nancy, FCRIN INI-CRCT, Nancy, France, ${ }^{2}$ Université de Lorraine, CNRS, Inria, LORIA, Nancy, France, ${ }^{3}$ Université \\ Paris-Saclay, CEA, Centre National de Recherche en Génomique Humaine, Evry, France, and ${ }^{4}$ Department of \\ Endocrinology, CHRU de Nancy, Nancy, France \\ Correspondence \\ should be addressed \\ to J P Ferreira \\ Email \\ j.ferreira@chru-nancy.fr
}

\begin{abstract}
Objective: Determining the factors associated with new-onset pre-diabetes and type 2 diabetes mellitus (T2D) is important for improving the current prevention strategies and for a better understanding of the disease. Design: To study the factors (clinical, circulating protein and genetic) associated with new onset pre-diabetes and T2D in an initially healthy (without diabetes) populational familial cohort with a long follow-up (STANISLAS cohort). Methods: A total of 1506 participants attended both the visit 1 and visit 4, separated by $\approx 20$ years. Over 400 proteins, GWAS and genetic associations were studied using models adjusted for potential confounders. Both prospective (V1 to V4) and cross-sectional (V4) analyses were performed.

Results: People who developed pre-diabetes $(n=555)$ and/or T2D $(n=73)$ were older, had higher BMI, blood pressure, glucose, LDL cholesterol, and lower eGFR. After multivariable selection, PAPP-A (pappalysin-1) was the only circulating protein associated with the onset of both pre-diabetes and T2D with associations persisting at visit 4 (i.e. $\approx 20$ years later). FGF-21 (fibroblast growth factor 21) was a strong prognosticator for incident T2D in the longitudinal analysis, but not in the cross-sectional analysis. The heritability of the circulating PAPP-A was estimated at 44\%. In GWAS analysis, the SNP rs634737 was associated with PAPP-A both at V1 and V4. External replication also showed lower levels of PAPP-A in patients with T2D.

Conclusions: The risk of developing pre-diabetes and T2D increases with age and with features of the metabolic syndrome. Circulating PAPP-A, which has an important genetic component, was associated with both the development and presence of pre-diabetes and T2D.
\end{abstract}

\section{Introduction}

The number of people with type 2 diabetes mellitus (T2D) more than doubled in the past 20 years in both the developed and developing countries (1). The latest prediction from the International Diabetes Federation is that over 380 million people are living with diabetes nowadays and that by 2035 this number may rise up to 600 million (2). Type 2 diabetes was traditionally considered as a disorder of middle-aged and elderly people; however, 
the more recent 'type 2 diabetes epidemics' is particularly worrisome among adolescents and young adults, leading to tremendous costs, impairment in the quality of life and reduced life expectancy, mainly due to associated cardiovascular and renal complications $(1,3,4)$.

Type 2 diabetes results from a complex interaction between genetic/epigenetic systems and multiple behavioral plus environmental factors (1). Despite the challenges linked to the gene-environment interplay, determining the factors associated with new-onset prediabetes and T2D is important not only for improving the current prevention strategies and treatments, but also for a better understanding of the disease $(5,6)$.

The STANISLAS (Suivi Temporaire Annuel NonInvasif de la Santé des Lorrains Assurés Sociaux) cohort is a single-centre familial prospective cohort comprising 1006 initially healthy (including absence of diabetes) families (4295 participants) from the Nancy region of France, who were recruited from 1993 to 1995 to the visit 1 (V1). Participants were then followed each 5 to 10 years, and from the initial visit, 1705 participants returned for the fourth visit (V4), held from 2011 to 2016, which comprised a detailed clinical and laboratorial assessment (7). Beyond the clinical, imaging and routine laboratorial measurements, circulating levels of multiple proteins were also assessed at V1 and V4. Additionally, a genome wide association study (GWAS) was performed for the V4 participants. This design allows the assessment of the clinical factors, genetic variants and proteomic signatures associated with the new-onset of pre-diabetes and/or T2D almost 20 years in advance; the family structure also allows the study of heritability (8).

In the present analysis, we considered patients without diabetes at V1 (patients entering the familial STANISLAS study did not have chronic conditions) with the main aims of studying the factors associated with new onset pre-diabetes and T2D at V4, considering the clinical features, proteomic signatures and genetic variants that might help explaining the main proteomic findings.

\section{Methods}

\section{Study population}

A detailed description of the STANISLAS cohort has been previously published (7). The STANISLAS cohort was established with the primary objective of investigating gene-gene and gene-environment interactions in the field of cardiovascular diseases. In order to assess the effect of genetics on the variability of intermediate phenotypes on the transition toward pathology, the families were deemed healthy and free of declared acute and/or chronic illness at V1. The implementation of the fourth visit (V4) enabled a follow-up of 18 to 23 years (mean of 18.5 years). The collected information was enriched with new circulating proteins and detailed clinical phenotyping. The study protocols for all examinations were reviewed and approved by the Local Ethics Committee of CPPEst 3, France. All participants provided written informed consent to participate in the study.

In the present analysis we included 1506 participants that attended both the V1 and V4 and in whom we had information on fasting glucose, random glucose, glycated hemoglobin, treatments and/or previous diagnosis that allow the pre-diabetes and T2D classification. The study flow-chart is shown in the Fig. 1. The main objective is to assess the circulating proteins that are associated with the development of diabetes in a prospective analysis (V1 to V4) and to ascertain if the associations remained significant almost 20 years later at V4 in a crosssectional analysis.

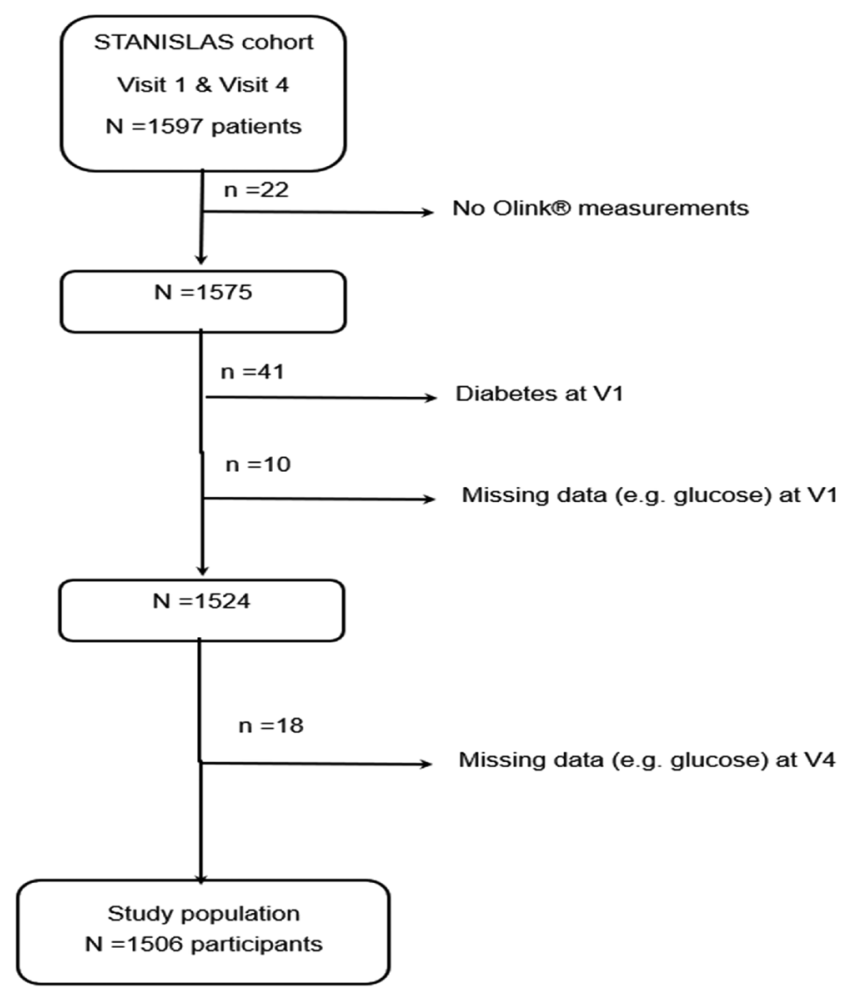

Figure 1

Study flow chart. 


\section{Study design}

All participants were observed at the Centre d'Investigation Clinique Plurithématique Pierre Drouin at Nancy Hospital Center (CIC-P de Nancy) in the morning after a 12- to 14-h fast. Blood samples were drawn, and medical history, medications, anthropometric parameters, blood pressure, carotid-femoral pulse-wave velocity (PWV), and carotid intima-media thickness (cIMT) were recorded.

\section{Pre-diabetes and diabetes definition}

Pre-diabetes was defined as a fasting plasma glucose (FPG) between $100 \mathrm{mg} / \mathrm{dL}$ and $125 \mathrm{mg} / \mathrm{dL}$ or a glycated hemoglobin between $5.7 \%$ and $6.4 \%$. Diabetes was defined as a FPG $\geq 126 \mathrm{mg} / \mathrm{dL}$ or a glycated hemoglobin $\geq 6.5 \%$ or a random plasma glucose $\geq 200 \mathrm{mg} / \mathrm{dL}$ with symptoms of hypoglycemia or previous diagnosis of diabetes or antiglycemic drug therapy (9).

\section{Circulating proteins and gene-candidate analysis}

All samples were collected at the CIC-P de Nancy with minimally traumatic venipuncture. Standardized sample handling procedures enabled the collection of serum and plasma (EDTA, heparin) as well as buffy coat fraction. Blood DNA of all the participants to the Stanislas V4 was extracted using Gentra Puregene Blood Kit (Qiagen) and stored at $-20^{\circ} \mathrm{C}$. Genotyping was conducted at CNRGH (Evry, France) using two chips: the Illumina Global Screening Array (GSA), which is composed of 687572 intronic and exonic markers, and the Illumina Exome Array, which is constituted of 244330 SNPs, mostly exonic. All blood-derived bio-samples are stored in a central biobank facility with temperatures between $-80^{\circ} \mathrm{C}$ and $-196^{\circ} \mathrm{C}$ (as required, e.g. $-196^{\circ} \mathrm{C}$ for plasma and serum).

Baseline plasma samples were analysed for proteins by the TATAA-biocenter using the Olink Proseek® Multiplex cardiovascular (CVD) II, III, cardiometabolic (CM), organdamage (OD) and inflammatory (INF) panels, using a proximity extension assay (PEA) technology (10), where 92 oligonucleotide-labelled antibody probe pairs per panel (total of $92 \times 5 ; n=460$ proteins) are allowed to bind to their respective targets in the sample in 96-well plate format. When binding to their correct targets, they give rise to new DNA amplicons with each ID-barcoding their respective antigens. The amplicons are subsequently quantified using a Fluidigm BioMark ${ }^{\mathrm{TM}} \mathrm{HD}$ real-time PCR platform. The platform provides $\log _{2}$-normalized protein expression (NPX) data.

The strategy for the genetic analyses of the present study was the following. First, we conducted GWAS on diabetes occurrence, fasting glycemia and glycated hemoglobin. Secondly, GWAS was conducted on the plasma protein(s) associated with both pre-diabetes and T2D in both analysis (i.e. longitudinal and crosssectional). Thereafter, the GWAS top-SNP was tested for association with study outcome (i.e. pre-diabetes, T2D, fasting glycemia, glycated hemoglobin and circulating PAPPA-A).

Our findings were replicated using the baseline data of the HOMAGE (Bioprofiling Response to Mineralocorticoid Receptor Antagonists for the Prevention of Heart Failure; ClinicalTrials.gov Identifier: NCT02556450) trial, a prospective trial that included 527 people at risk for developing heart failure randomized to either spironolactone or standard of care for up to 9 months; the description of this study and baseline characteristics of the participants have been published elsewhere (11).

\section{Statistical, genetic and bioinformatical analysis}

For the baseline clinical characteristics, continuous variables are expressed as means and respective S.D. and compared using ANOVA or Kruskal-Wallis test. Categorical variables are presented as frequencies and percentages and compared using Chi-squared test.

The main aim of this study was to test the association of multiple circulating proteins with incident pre-diabetes and T2D. Multinomial logistic regression models were performed (using no pre-diabetes/T2D as the referent category). First, a stepwise backward model including all the clinical variables with a $P<0.1$ from Table 1 was performed, to select the clinical features with stronger association with pre-diabetes and T2D. Due to collinearity between BMI and waist circumference (correlation $>0.9$ in both visits), we included only BMI in the models (including waist circumference provided the same results). The clinical features retained in the final model were age, BMI, LDL cholesterol and systolic blood pressure. These variables were used for adjustment in all the subsequent models. Secondly, the potential association of the multiple circulating proteins with pre-diabetes and $\mathrm{T} 2 \mathrm{D}$ was tested one-by-one on top of the clinical model (i.e. adjusted for the clinical features) built in the previous step. The statistical significance level was fixed at a FDRq $<0.05$, after applying a Benjamini-Hochberg correction for multiple testing (i.e. false discovery rate (FDR) set at 5\%). 
มูร

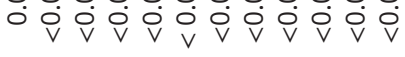

๙

0.

m.

m $m$ 밈

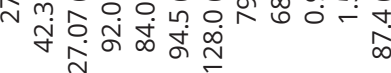

\section{$\stackrel{5}{>}$}

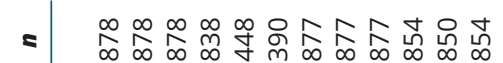

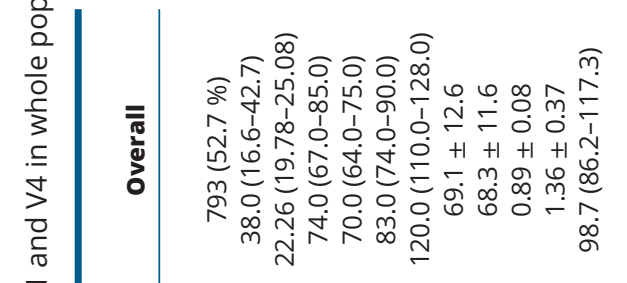

$=$

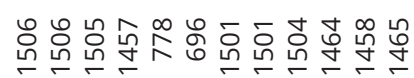

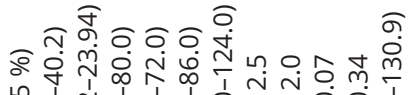
ñ

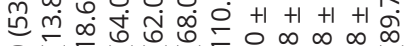

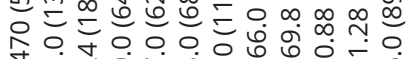

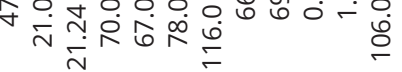

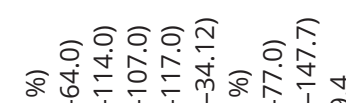

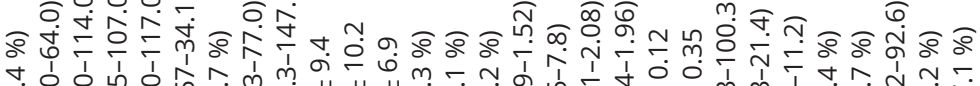

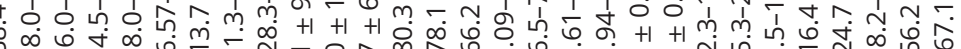
m N

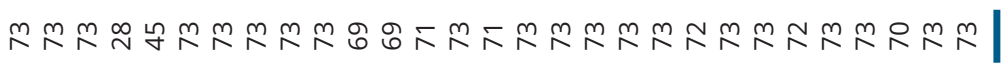

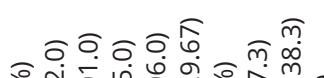
๙

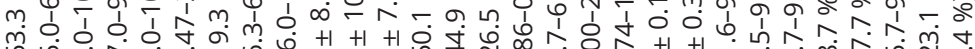
包出

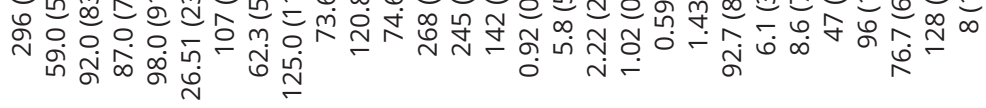

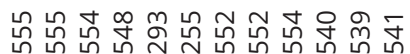

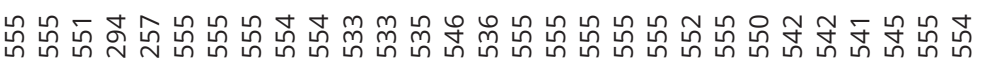

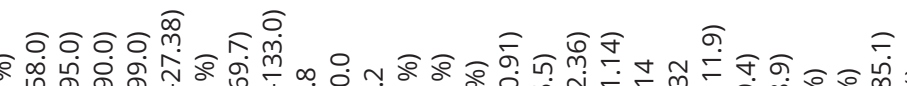

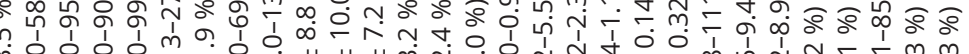
ñ م

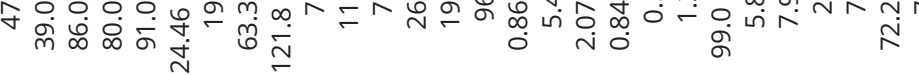

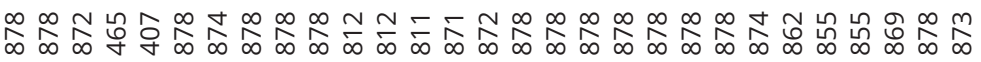
oิ oิ 0 oิ ○ ơ ن n్

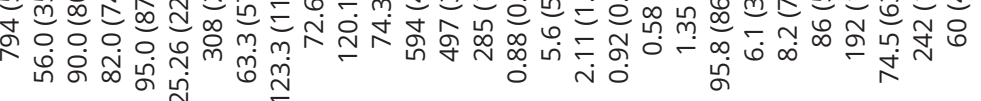

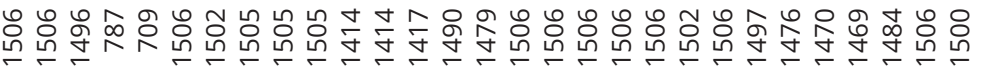
in

$\widehat{\underline{E}}$<smiles>C1CCCC1</smiles>

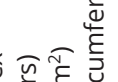

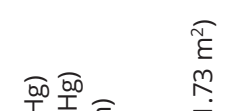

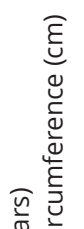

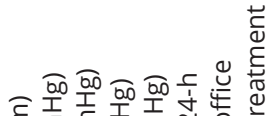
峁

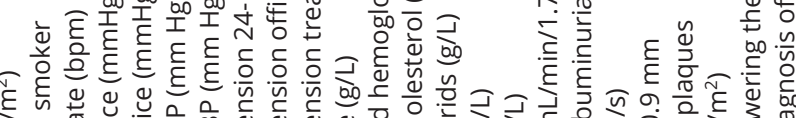
है है

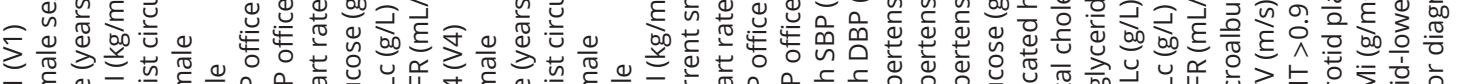

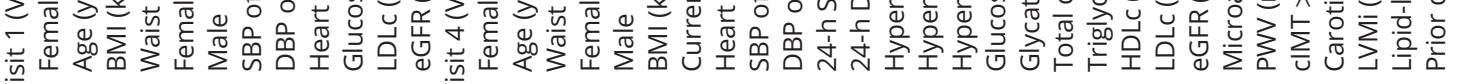
䓛 
Subsequently, the proteins selected at the 5\%FDR went through a multivariable stepwise forward selection model adjusted for the clinical variables. Since the circulating proteins were measured using NPX values on a $\log _{2}$ scale, the odds ratio (OR) for each protein estimates the increase/decrease in the odds of pre-diabetes/T2D associated with a doubling in the protein concentration. As exploratory analysis, we have assessed the additive value of the biomarkers on top of the clinical model for the 'prediction' of pre-diabetes and T2D onset (from V1 to V4). These analyses were performed using SAS version 9.1.

The genetic analyses were performed using R (version 3.4.1). GWAS were run using a linear mixed model, in order to take into account for the pedigree data, with age and sex used as covariates, the statistical significance level was fixed at $10^{-7}$. For heritability estimation, matrix of pedigree or genetic relationship were used in a linear mixed model to estimate the variance captured by additive genetic effects via average information restricted maximum likelihood analysis. To take into account the genetic information at both V1 and V4, we have added two random effects in the model (one for individuals and another for visits) in order to assess the variance component resulting from the repeated measures. Both models were implemented in the R package 'gaston'. For the top-SNP association tests, age and sex were used as covariates and the statistical significance level was fixed at 0.05 .

The complex network analysis was performed using the FHF-GKB (Fight Heart Failure - Graph Knowledge Box) resource, representing most available public knowledge about human protein-protein and protein-pathway relationships in a customized upgraded version of the EdgeBox provided by the EdgeLeap company (available from: https://www.edgeleap.com). FHF-GKB data is extracted from public data sources resulting in 20431 protein nodes imported from Uniprot (13), including all proteins involved in this study and 2272 pathway nodes from Reactome (v69)1 and 47162 disease nodes from Disease Ontology and Human Phenotype Ontology (12). Protein-protein relationships were retrieved from STRING (v11) (15) or Reactome or WikiPathways (16) or Mentha (17) or BioGrid (18), protein-pathway relationships from Reactome and protein-disease relationships from DisGeNET (13). The FHF-GKB complex network was queried in order to explore pathways and proteins that connect the circulating proteins to disease through common mechanistic pathways. Queries were expressed according to patterns defining a path structure between two nodes, such as 'protein-disease' and 'pathway-protein-disease'. The resulting graph is displayed in figure illustrating the main connection between proteins, pathways, and disease (i.e. T2D (HP:0005978)).

\section{Results}

\section{Patient characteristics}

The patients' characteristics at V1 overall $(n=1506)$ and by the pre-diabetes $(n=555 ; 37 \%)$ and T2D $(n=73 ; 4.8 \%)$ at V4 are depicted in the Table 1 . Patients who developed pre-diabetes and/or T2D were older, had higher BMI, blood pressure, glucose levels, LDL cholesterol, and lower estimated glomerular filtration rate (eGFR).

\section{Clinical factors associated with pre-diabetes and type 2 diabetes mellitus}

The clinical factors independently associated with prediabetes onset were older age and higher LDL cholesterol and with T2D were older age, higher BMI, higher systolic blood pressure, and higher LDL cholesterol (tendency) (Table 2).

\section{Circulating proteins associated with diabetes}

The full list of proteins, their respective associations with pre-diabetes and T2D, and details on the Olink ${ }^{\circledR}$ technology are depicted in the Supplementary material (see section on supplementary materials given at the end of this article).

After multivariable selection adjusted for the clinical features, PAPP-A (pappalysin-1) was the only circulating protein that was associated with lower odds of both prediabetes and $\mathrm{T} 2 \mathrm{D}$ onset in the V1 to V4 (prospective) analysis and also with the presence of pre-diabetes and T2D at V4 (cross-sectional analysis). CALC-A (calcitonin related polypeptide alpha) and VCAM-1 (vascular cell adhesion molecule 1) were also associated with lower odds of developing pre-diabetes (but not diabetes) in the V1 to V4 prospective analysis, but the associations were no longer present at the V4 cross-sectional analysis. On the other hand, FABP2 (fatty acid binding protein 2), LOX-1 (lysyl oxidase), and THPO (thrombopoietin) were associated with higher odds of developing pre-diabetes (but not diabetes) from V1 to V4, whereas FGF-21 (fibroblast growth factor 21), MCP-4 (mast-cell protease 4), SERPINA-12 (serpin family A member 12), and VSIG-2 (V-set and immunoglobulin domain containing 2) were associated with higher odds of developing diabetes from 
V1 to V4. None of these associations remained significant at V4. The details of the associations are fully depicted in the Table 3. In the external replication analysis, patients with diabetes prospectively recruited in the HOMAGE clinical trial also presented lower levels of PAPP-A (Supplementary Table 1).

In the exploratory 'predictive' analysis, the clinical model for prediabetes presented an AUC of 0.74, and adding PAPP-A to this model improved its predictive capacity to 0.75 ( $P$-value for AUC comparison=0.003). The clinical model performed even better for diabetes with an AUC of 0.90, and adding PAPP-A to this model did not improve its predictive capacity as the AUC remained at 0.90 ( $P$-value for AUC comparison=0.32). However, adding FGF-21 (that had a strong association with new-onset diabetes) improved this model to an AUC of 0.92 ( $P$-value for AUC comparison=0.020). Adding

Table 2 Multinomial logistic regression: clinical predictors at visit 1 (V1) of pre-diabetes (Pre-DM) and diabetes (DM) at visit 4 (V4) (prospective analysis), and association of V4 clinical variables for Pre-DM and DM (cross-sectional analysis).

\begin{tabular}{|c|c|c|}
\hline Risk factor/Status & OR $(95 \% \mathrm{Cl})$ & P-value \\
\hline \multicolumn{3}{|c|}{ V1 clinical predictors at V4 (prospective) } \\
\hline \multicolumn{3}{|c|}{ Age per 10 years } \\
\hline Pre-DM & $1.87(1.67-2.10)$ & $<0.0001$ \\
\hline DM & $2.07(1.45-2.95)$ & $<0.0001$ \\
\hline \multicolumn{3}{|l|}{ BMI per 1 kg/m² } \\
\hline Pre-DM & $1.03(0.99-1.07)$ & 0.12 \\
\hline DM & $1.24(1.15-1.33)$ & $<0.0001$ \\
\hline \multicolumn{3}{|l|}{$\mathrm{LDL} \geq 1.6 \mathrm{~g} / \mathrm{L}$} \\
\hline Pre-DM & $1.45(1.09-1.92)$ & 0.011 \\
\hline DM & $1.69(0.97-2.96)$ & 0.065 \\
\hline \multicolumn{3}{|c|}{ SBP per $10 \mathrm{mmHg}$} \\
\hline Pre-DM & $0.99(0.89-1.10)$ & 0.84 \\
\hline DM & $1.44(1.15-1.80)$ & 0.001 \\
\hline \multicolumn{3}{|c|}{ V4 clinical variables (cross-sectional) } \\
\hline \multicolumn{3}{|c|}{ Age per 10 years } \\
\hline Pre-DM & $2.03(1.82-2.25)$ & $<0.0001$ \\
\hline DM & $2.62(1.91-3.60)$ & $<0.0001$ \\
\hline \multicolumn{3}{|l|}{$\mathrm{HR} \geq 80 \mathrm{bpm}$} \\
\hline Pre-DM & $0.79(0.42-1.47)$ & 0.46 \\
\hline DM & 3.35 (1.49-7.57) & 0.004 \\
\hline \multicolumn{3}{|l|}{$\mathrm{BMI}$ per $1 \mathrm{~kg} / \mathrm{m}^{2}$} \\
\hline Pre-DM & $1.07(1.04-1.10)$ & $<0.0001$ \\
\hline DM & $1.19(1.13-1.25)$ & $<0.0001$ \\
\hline \multicolumn{3}{|l|}{ LDLC $\geq 1.6 \mathrm{~g} / \mathrm{L}$} \\
\hline Pre-DM & $1.30(0.99-1.70)$ & 0.057 \\
\hline DM & $0.26(0.11-0.63)$ & 0.003 \\
\hline \multicolumn{3}{|c|}{ SBP per $10 \mathrm{mmHg}$} \\
\hline Pre-DM & $0.94(0.86-1.02)$ & 0.13 \\
\hline $\mathrm{DM}$ & $1.19(1.01-1.40)$ & 0.036 \\
\hline
\end{tabular}

$\mathrm{BMI}$, body mass index; $\mathrm{HR}$, heart rate; $\mathrm{LCLC}$, low density lipoprotein cholesterol; OR, odds ratio; SBP, systolic blood pressure (office). more biomarkers lead only to marginal improvements in the models.

\section{Genetic associations, heritability estimation, and complex network analyses}

No signal was found for GWAS with diabetes, fasting glycemia or glycated hemoglobin. For circulating PAPP-A, the heritability was estimated at $44 \%$ for subjects with measurements at both visits (V1 and V4) (Supplementary Table 2). Only one SNP, rs634737, was associated with the protein PAPP-A both at V1 and V4 at the GWAS significance level (Table 4). Association tests between this SNP and study outcome are shown in the Table 5 and suggests that subjects with mutation at rs634737 are more susceptible to develop diabetes, have higher level of fasting glycemia and glycated hemoglobin.

Network analysis results are presented in the Fig. 2.

\section{Discussion}

Our study shows that the risk of developing pre-diabetes and T2D increases with age and with features of the metabolic syndrome. Furthermore, several circulating proteins were associated with pre-diabetes and T2D; particularly, PAPP-A was associated with the development of both pre-diabetes and T2D (i.e. in initially healthy young adults without diabetes, at $\mathrm{V} 1 \approx 19$ years before V4) and remained associated with both pre-diabetes and T2D once the disease had been installed (at V4). In this familial cohort, the PAPP-A protein has an important heritability $(\approx 44 \%)$ and acts via proteins and a pathway linked to IGFs. These data were externally replicated in the HOMAGE trial cohort and provide novel insights on the pathophysiology of T2D, generating hypothesis for future research in the field.

Beyond the genetic susceptibility, that may be particularly important in specific ethnic groups $(14,15,16)$, T2D is essentially caused by an interplay between environmental and behavioral factors that include sedentary lifestyle, nutritional habits rich in sugars, carbohydrates and processed foods, and obesity (17). The lack of robust genetic associations with diabetes in our study also supports this notion. Importantly, it has been suggested that the risk of dysglycemia-associated complications commences many years before the onset of clinical diabetes (18). Our findings also support that the metabolic alterations start many years before the onset of T2D and are also associated with pre-diabetes, moreover 
Table 3 Final protein selection at V1 for the prediction of pre-diabetes and diabetes at V4 (prospective analysis) and V4 final protein selection for the prediction of pre-diabetes and diabetes at V4 (cross-sectional analysis).

\begin{tabular}{|c|c|c|c|}
\hline Variable & Status & Adjusted *OR $(95 \% \mathrm{Cl})$ & P-value \\
\hline \multicolumn{4}{|c|}{ Visit 1 (V1) proteomic predictors of pre-diabetes and diabetes at V4 (prospective) } \\
\hline \multirow[t]{2}{*}{ PAPP-A } & Pre-DM & $0.71(0.55-0.92)$ & 0.008 \\
\hline & DM & $0.54(0.30-0.96)$ & 0.036 \\
\hline \multirow[t]{2}{*}{ CALCA } & Pre-DM & $0.79(0.66-0.94)$ & 0.007 \\
\hline & DM & $1.20(0.82-1.76)$ & 0.36 \\
\hline \multirow[t]{2}{*}{ VCAM1 } & Pre-DM & $0.60(0.42-0.87)$ & 0.007 \\
\hline & DM & $0.77(0.35-1.70)$ & 0.52 \\
\hline \multirow[t]{2}{*}{ FABP2 } & Pre-DM & $1.25(1.05-1.50)$ & 0.013 \\
\hline & $\mathrm{DM}$ & $0.82(0.53-1.27)$ & 0.37 \\
\hline \multirow[t]{2}{*}{ LOX-1 } & Pre-DM & 1.49 (1.15-1.93) & 0.002 \\
\hline & DM & $1.06(0.56-2.02)$ & 0.86 \\
\hline \multirow[t]{2}{*}{ THPO } & Pre-DM & $1.88(1.16-3.04)$ & 0.01 \\
\hline & $\mathrm{DM}$ & $0.73(0.24-2.21)$ & 0.58 \\
\hline \multirow[t]{2}{*}{ FGF-21 } & Pre-DM & $1.07(0.97-1.18)$ & 0.19 \\
\hline & DM & $1.83(1.47-2.30)$ & $<0.0001$ \\
\hline \multirow[t]{2}{*}{ MCP-4 } & Pre-DM & $1.08(0.84-1.39)$ & 0.54 \\
\hline & $\mathrm{DM}$ & $2.28(1.31-3.99)$ & 0.004 \\
\hline \multirow[t]{2}{*}{ SERPINA12 } & Pre-DM & $1.01(0.92-1.12)$ & 0.81 \\
\hline & $\mathrm{DM}$ & 1.39 (1.09-1.76) & 0.008 \\
\hline \multirow[t]{2}{*}{ VSIG2 } & Pre-DM & $1.16(0.83-1.63)$ & 0.38 \\
\hline & $\mathrm{DM}$ & $2.68(1.27-5.65)$ & 0.009 \\
\hline \multicolumn{4}{|c|}{ Visit 4 (V4) proteomic associations with pre-diabetes and diabetes (cross-sectional) } \\
\hline \multirow[t]{2}{*}{ PAPP-A } & Pre-DM & $0.57(0.44-0.74)$ & $<0.0001$ \\
\hline & DM & $0.50(0.25-0.99)$ & 0.048 \\
\hline \multirow[t]{2}{*}{ EPHB4 } & Pre-DM & $0.39(0.19-0.78)$ & 0.008 \\
\hline & DM & $0.15(0.02-0.97)$ & 0.046 \\
\hline \multirow[t]{2}{*}{ CES2 } & Pre-DM & $3.27(1.78-6.00)$ & 0.0001 \\
\hline & DM & $3.12(1.12-8.64)$ & 0.029 \\
\hline \multirow[t]{2}{*}{ CD5 } & Pre-DM & $1.99(1.26-3.14)$ & 0.003 \\
\hline & $\mathrm{DM}$ & $3.47(1.19-10.1)$ & 0.023 \\
\hline \multirow[t]{2}{*}{ GDNF } & Pre-DM & $1.51(1.11-2.05)$ & 0.008 \\
\hline & DM & $2.56(1.51-4.35)$ & 0.0005 \\
\hline \multirow[t]{2}{*}{ REN } & Pre-DM & $1.21(0.99-1.48)$ & 0.057 \\
\hline & DM & $1.70(1.06-2.75)$ & 0.028 \\
\hline \multirow[t]{2}{*}{ SCGB3A2 } & Pre-DM & $1.35(1.12-1.62)$ & 0.001 \\
\hline & $\mathrm{DM}$ & $1.54(1.1-2.178)$ & 0.012 \\
\hline CD8A & Pre-DM & $0.68(0.55-0.85)$ & 0.0008 \\
\hline & $\mathrm{DM}$ & $0.65(0.36-1.16)$ & 0.15 \\
\hline CX3CL1 & Pre-DM & $0.41(0.26-0.66)$ & 0.0002 \\
\hline & DM & $0.36(0.09-1.35)$ & 0.13 \\
\hline PRSS8 & Pre-DM & $2.12(1.40-3.22)$ & 0.0004 \\
\hline & $\mathrm{DM}$ & $2.52(0.75-8.44)$ & 0.13 \\
\hline TRAIL & Pre-DM & $2.19(1.30-3.68)$ & 0.003 \\
\hline & DM & $2.74(0.70-10.6)$ & 0.14 \\
\hline ENTPD2 & Pre-DM & $1.20(0.70-2.06)$ & 0.5 \\
\hline & DM & $0.09(0.01-0.44)$ & 0.003 \\
\hline KLK6 & Pre-DM & $0.65(0.36-1.16)$ & 0.15 \\
\hline & $\mathrm{DM}$ & $0.02(0.01-0.10)$ & $<0.0001$ \\
\hline MCP2 & Pre-DM & $1.19(0.95-1.41)$ & 0.12 \\
\hline & DM & $0.40(0.22-0.73)$ & 0.003 \\
\hline AIFM1 & Pre-DM & $1.00(0.87-1.15)$ & 0.97 \\
\hline & $\mathrm{DM}$ & $1.63(1.14-2.32)$ & 0.007 \\
\hline AOC3 & Pre-DM & $1.38(0.96-1.96)$ & 0.074 \\
\hline & $\mathrm{DM}$ & $2.77(1.08-7.07)$ & 0.033 \\
\hline DPP6 & Pre-DM & $1.49(0.95-2.32)$ & 0.079 \\
\hline & DM & $12.4(3.67-42.3)$ & $<0.0001$ \\
\hline
\end{tabular}


Table 3 Continued.

\begin{tabular}{cll}
\hline Variable & Status \\
\hline GDF-15 & Pre-DM \\
IL-1RT2 & DM \\
& Pre-DM \\
MMP-10 & DM \\
& Pre-DM \\
& DM \\
\hline
\end{tabular}

\begin{tabular}{llc}
\hline Adjusted *OR $(95 \% \mathrm{Cl})$ & & P-value \\
\hline $1.02(0.69-1.51)$ & & 0.89 \\
$12.3(5.46-27.8)$ & $<0.0001$ \\
$1.04(0.65-1.66)$ & 0.86 \\
$6.13(1.94-19.4)$ & 0.002 \\
$1.01(0.79-1.26)$ & 0.96 \\
$2.98(1.65-5.39)$ & 0.0003 \\
\hline
\end{tabular}

Proteins ordered from top to bottom by their association with both pre-diabetes and diabetes (first), then pre-diabetes alone (second), and finally diabetes alone (third); following this order proteins that are associated with a lower risk of diabetes appear first.

*Adjusted for age, BMI, LDL cholesterol and systolic blood pressure in a stepwise forward model (see also the Table 2).

PAPP-A, pappalysin 1 (the only common protein between V1 and V4 and that is associated with lower risk of pre-diabetes and diabetes at both V1 and V4); EPHB4, EPH receptor B4; CES2, carboxylesterase 2; CD5, CD5 T-cell surface glycoprotein; GDNF; glial cell derived neurotrophic factor; REN, renin; SCGB3A2, secretoglobin family 3A member 2; CD8A, T-cell surface glycoprotein CD8 alpha chain; CX3CL1, C-X3-C motif chemokine ligand 1; PRSS8, serine protease 8; TRAIL, TNF superfamily member 10; ENTPD2, ectonucleoside triphosphate diphosphohydrolase 2; KLK6, kallikrein related peptidase 6; MCP2, macrophage cationic peptide 2; AIFM1, apoptosis inducing factor mitochondria associated 1; AOC3, amine oxidase copper containing 3; DPP6, dipeptidyl peptidase like 6; GDF-15, growth differentiation factor 15; IL-1RT2, interleukin 1 receptor type 2; MMP-10, matrix metalloproteinase 10; CALCA, calcitonin related polypeptide alpha; VCAM1, vascular cell adhesion molecule 1; FABP2, fatty acid binding protein 2; LOX-1, lysyl oxidase; THPO, thrombopoietin; FGF-21, fibroblast growth factor 21; MCP-4, mast-cell protease 4; SERPINA12, serpin family A member 12; VSIG2, V-set and immunoglobulin domain containing 2.

the proteomic profiles of these patients are altered (17, $19,20,21)$. Landmark clinical trials have shown that lifestyle modification programs focused on weight loss and/or the use of metformin (although the lifestyle interventions aiming at significant weight loss were the most effective measure) can delay the onset of T2D (22, $23,24)$. Unfortunately, no study has shown that diabetes can be delayed or prevented in a community setting (19). This should not demotivate us from continuing the efforts and possibly aiming the preventive strategies to individuals at higher risk for developing the disease may be more effective than an one-size-fits-all strategy (25). In this regard, people who developed pre-diabetes and T2D had lower levels of circulating PAPP-A (compared to those who did not developed pre-diabetes nor diabetes) and continued expressing lower levels of this protein after the disease was already installed. These findings were replicated in patients prospectively recruited for the HOMAGE trial, where those with diabetes also expressed lower levels of PAPP-A. PAPP-A increases the IGFs bioavailability and stimulates IGF-mediated growth through cleavage of the insulin-like growth factor binding protein-4 (IGFBP-4) (26). The two central components of the IGF system, IGF-I and IGF-II, serve as ligands for the ubiquitously expressed IGF-I receptor, through which they facilitate cellular proliferation and differentiation as well as metabolic effects mimicking those conveyed by insulin (27). Our complex network analyses support that PAPP-A interacts directly with IGF I and II through a pathway linked to IGFs. High concentrations of PAPP-A were initially found in women during pregnancy, but later PAPP-A was found to be the primary IGFBP-4 protease in humans and many other species $(28,29,30)$. Under normal circumstances, IGFBPs bind IGFs with high affinity, stabilize the IGFs and transport them to peripheral tissues, where proteases, such as PAPP-A, cleave IGFBPs into low binding-affinity fragments to allow release and action of IGFs. Thus, PAPP-A actions occur within the tissues, and

Table 4 GWAS associations with circulating levels of PAPP-A.

\begin{tabular}{l} 
SNP id \\
\hline Visit 1 (V1) \\
rs634737 \\
rs61806064 \\
rs75242093 \\
rs62412362 \\
rs72630775 \\
Visit 4 (V4) \\
rs634737 \\
rs11606592 \\
rs10400305
\end{tabular}

\begin{tabular}{lllll} 
A1 & & A2 & & freqA2 \\
\cline { 1 - 1 } & & & & \\
$A$ & & $G$ & & 0.732 \\
$A$ & & $G$ & & 0.990 \\
$A$ & & $G$ & & 0.970 \\
$A$ & & $G$ & & 0.915 \\
$A$ & & $G$ & & 0.992 \\
& & & \\
$A$ & & $G$ & & 0.734 \\
$C$ & & $T$ & & 0.898 \\
$G$ & & & 0.843
\end{tabular}

$\begin{array}{r}\hline \multicolumn{1}{c}{\text { beta }} \\ \hline 0.147 \\ -0.588 \\ -0.342 \\ -0.199 \\ -0.630 \\ 0.160 \\ -0.204 \\ -0.149 \\ \hline\end{array}$

\begin{tabular}{c}
\hline s.D. \\
\hline 0.024 \\
0.102 \\
0.060 \\
0.037 \\
0.116 \\
0.022 \\
0.032 \\
0.027 \\
\hline
\end{tabular}

\begin{tabular}{|c|c|}
\hline P-value* & chr \\
\hline 9.02E-10 & 11 \\
\hline 8.37E-09 & 1 \\
\hline $1.53 \mathrm{E}-08$ & 4 \\
\hline 5.19E-08 & 6 \\
\hline $6.12 \mathrm{E}-08$ & 7 \\
\hline $9.14 \mathrm{E}-13$ & 11 \\
\hline $2.03 \mathrm{E}-10$ & 11 \\
\hline $2.18 \mathrm{E}-08$ & 11 \\
\hline
\end{tabular}

*Age and sex are used as covariates; statistical significance has been set at $10^{-7}$. 
Table 5 Association test between rs634737 and diabetes, pre-diabetes and associated continuous outcomes.

\begin{tabular}{|c|c|c|}
\hline & Beta *( \pm S.D. $)$ & $P$ value \\
\hline Diabetes & $0.056(0.027)$ & 0.04 \\
\hline Pre-diabetes & $0.014(0.019)$ & 0.45 \\
\hline Fasting glycemia & $0.015(0.006)$ & 0.02 \\
\hline Glycated hemoglobin & $0.056(0.022)$ & 0.01 \\
\hline
\end{tabular}

*Risk per minor allele, adjustment for sex and age.

the direct liberation of IGF is a local event. PAPP-A could be a modulator of the local action of IGF-1, and increased PAPP-A levels may reflect a repair mechanism against vascular damage (31). Collectively, whether glucose, insulin or both that affect PAPP-A/IGFBP-4 levels and the clinical meaning of the circulating levels of PAPP-A remain speculative $(32,33)$. The association of PAPP-A with diabetes in clinical studies has also been inconsistent $(33,34,35)$. It has been reported that genetic deletion of PAPP-A in mice confers resistance to the development of diabetic nephropathy and induces an overall reduction in age-related diseases $(36,37,38)$. The human PAPP-A gene is located on chromosome 9 (39). In the present study, the SNP rs634737 was strongly associated with the expression of PAPP-A both at V1 and V4 and may provide valuable information for future studies on the role of PAPP-A. Moreover, this SNP was also associated with T2D, fasting glycemia, and glycated hemoglobin; thus, reinforcing the strength of our findings. Collectively, given that experimental and clinical data on PAPP-A

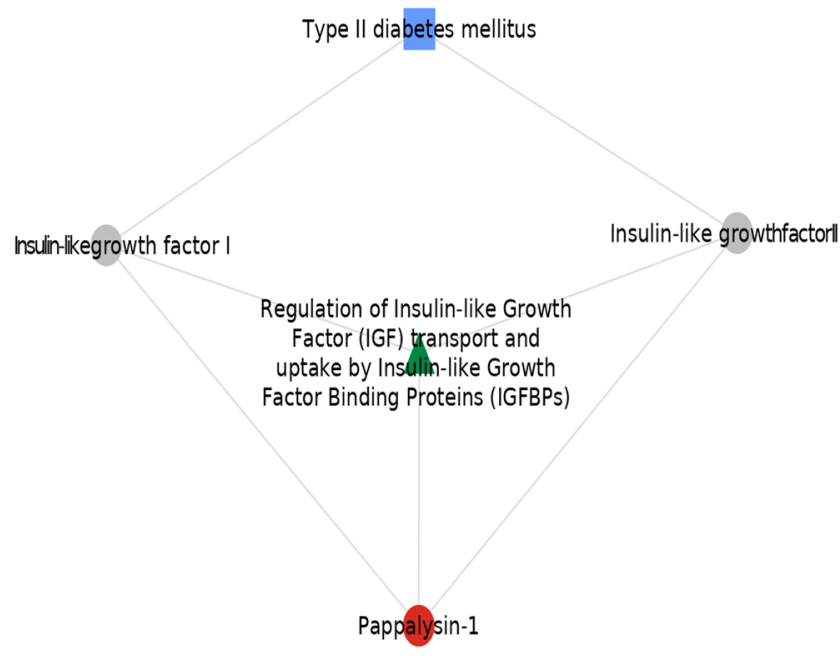

\section{Figure 2}

Pathway (in green) and proteins (grey circles) linking PAPP-A (red circle) to diabetes (blue square). A full colour version of this figure is available at https://doi.org/10.1530/EJE-20-0246. have been rather inconclusive so far, much remains to be elucidated about their roles in metabolic diseases and complications (26).

Other proteins were also associated with the onset and presence of pre-diabetes and T2D, and these may represent several pathways associated with obesity, glucose intolerance, inflammation, apoptosis, angiogenesis and oxidative stress $(40,41,42,43)$. Particularly, FGF-21 had a strong association with new-onset diabetes later in life. FGF-21 is an endocrine hormone that regulates energy homeostasis and insulin sensitivity (44). Patients with T2D may present higher circulating levels of FGF-21, which may be influenced by other metabolic factors such as obesity and cholesterol (45). In our study, adding FGF21 to the clinical model for predicting diabetes improved this model which already had a very good performance with the clinical variables alone. However, a causal link between the levels of FGF-21 and T2D has not been established (46). The proteins (e.g. EPHB4, VCAM1, CES2) were only present in either one of the visits.

\section{Limitations}

Some limitations and caution in the interpretation of the results should be acknowledged in the present study. First, this is an observational study, therefore no causality can be established and these findings should be regarded as hypothesis generating. Second, PAPP-A and other circulating proteins were measured with the Olink® technology standardized $\log _{2}$ NPX values (as described in the methods section), hence no direct conversion to the standard values is possible. Fourth, the SNP rs634737 is located in a non-coding region of the genome; therefore, no conclusion can be drawn about its mode of action and no replication could be performed and these results should be regarded with caution. Fifth, Mendelian randomization analyses could not be performed due to the our 'small' sample size for this type of analysis.

\section{Conclusion}

The risk of developing pre-diabetes and T2D increases with age and with features of the metabolic syndrome. Several circulating proteins were associated with pre-diabetes and T2D. Particularly, circulating PAPP- A, which has an important genetic component, was associated with both the development and presence of pre-diabetes and T2D. Our data provide novel insights on the pathophysiology 
of T2D and generate hypothesis for future research in the field, including the study of PAPP-A as a potential biotarget for the prevention and/or early treatment of diabetes.

\section{Supplementary materials}

This is linked to the online version of the paper at https://doi.org/10.1530/ EJE-20-0246.

\section{Declaration of interest}

The authors declare that there is no conflict of interest that could be perceived as prejudicing the impartiality of this study.

\section{Funding}

The STANISLAS study is sponsored by the CHRU de NANCY. This work is supported by the French Ministry of Health 'Programme Hospitalier de Recherche Clinique inter regional 2013', by the Contrat de Plan EtatLorraine, and a public grant overseen by the French National Research Agency (ANR) as part of the second 'Investissements d'Avenir' program (reference: ANR-15-RHUS-0004). It is also supported by the European FibroTargets Project (grant agreement No. SP7\#602904), European HOMAGE project (grant agreement $\mathrm{N}^{\circ}$ Heart 'Omics' in Ageing, 7th Framework Program grant \# 305507), the MEDIA project (Européen 'Cooperation' - Theme "Health" / FP7-HEALTH-2010-single-stage (reference: 261409.), FIGHT-HF (reference: ANR-15-RHU-0004), FOCUS-MR (reference: ANR-15CE14-0032-01), ERA-CVD EXPERT (reference: ANR-16-ECVD-0002-02) and la Fondation de Recherche en Hypertension Artérielle.

\section{References}

1 Chen L, Magliano DJ \& Zimmet PZ. The worldwide epidemiology of type 2 diabetes mellitus - present and future perspectives. Nature Reviews: Endocrinology 20118 228-236. (https://doi.org/10.1038/ nrendo.2011.183)

2 Whiting DR, Guariguata L, Weil C \& Shaw J. IDF diabetes atlas: global estimates of the prevalence of diabetes for 2011 and 2030. Diabetes Research and Clinical Practice 201194 311-321. (https://doi. org/10.1016/j.diabres.2011.10.029)

3 NCD Risk Factor Collaboration (NCD-RisC). Worldwide trends in diabetes since 1980: a pooled analysis of 751 population-based studies with 4.4 million participants. Lancet 2016387 1513-1530. (https://doi.org/10.1016/S0140-6736(16)00618-8)

4 Zhang P, Zhang X, Brown J, Vistisen D, Sicree R, Shaw J \& Nichols G. Global healthcare expenditure on diabetes for 2010 and 2030. Diabetes Research and Clinical Practice 201087 293-301. (https://doi. org/10.1016/j.diabres.2010.01.026)

5 Saha S, Gerdtham UG \& Johansson P. Economic evaluation of lifestyle interventions for preventing diabetes and cardiovascular diseases. International Journal of Environmental Research and Public Health 20107 3150-3195. (https://doi.org/10.3390/ijerph7083150)

6 Backholer K, Peeters A, Herman WH, Shaw JE, Liew D, Ademi Z \& Magliano DJ. Diabetes prevention and treatment strategies: are we doing enough? Diabetes Care 201336 2714-2719. (https://doi. org/10.2337/DC12-2501)

7 Ferreira JP, Girerd N, Bozec E, Merckle L, Pizard A, Bouali S, Eby E, Leroy C, Machu JL, Boivin JM et al. Cohort Profile: rationale and design of the fourth visit of the Stanislas cohort: a familial longitudinal population-based cohort from the Nancy region of France. International Journal of Epidemiology 201847 395-395j. (https://doi.org/10.1093/ije/dyx240)
8 Xhaard C, Dandine-Roulland C, Villemereuil P, Floch EL, BacqDaian D, Machu JL, Ferreira JP, Deleuze JF, Zannad F, Rossignol P et al. Heritability of a resting heart rate in a 20-year follow-up family cohort with GWAS data: insights from the Stanislas cohort. European Journal of Preventive Cardiology 2019 In press. (https://doi. org/10.1177/2047487319890763)

9 American Diabetes Association. 2. Classification and diagnosis of diabetes: standards of medical care in Diabetes-2019. Diabetes Care 201942 (Supplement 1) S13-S28. (https://doi.org/10.2337/dc19-S002)

10 Lundberg M, Eriksson A, Tran B, Assarsson E \& Fredriksson S. Homogeneous antibody-based proximity extension assays provide sensitive and specific detection of low-abundant proteins in human blood. Nucleic Acids Research 201139 e102. (https://doi.org/10.1093/ nar/gkr424)

11 Pellicori P, Ferreira JP, Mariottoni B, Brunner-La Rocca HP, Ahmed FZ, Verdonschot J, Collier T, Cuthbert JJ, Petutschnigg J, Mujaj B et al. Effects of spironolactone on serum markers of fibrosis in people at high risk of developing heart failure: rationale, design and baseline characteristics of a proof-of-concept, randomised, precision-medicine, prevention trial. The Heart OMics in AGing (HOMAGE) trial. European Journal of Heart Failure 2020 In press. (https://doi.org/10.1002/ejhf.1716)

12 Kohler S, Carmody L, Vasilevsky N, Jacobsen JOB, Danis D, Gourdine JP, Gargano M, Harris NL, Matentzoglu N, McMurry JA et al. Expansion of the Human Phenotype Ontology (HPO) knowledge base and resources. Nucleic Acids Research 201947 D1018-D1027. (https://doi.org/10.1093/nar/gky1105)

13 Pinero J, Bravo À, Queralt-Rosinach N, Gutierrez-Sacristan A, DeuPons J, Centeno E, Garcia-Garcia J, Sanz F \& Furlong LI. DisGeNET: a comprehensive platform integrating information on human disease-associated genes and variants. Nucleic Acids Research 201745 D833-D839. (https://doi.org/10.1093/nar/gkw943)

14 Franco M, Bilal U, Ordunez P, Benet M, Morejon A, Caballero B, Kennelly JF \& Cooper RS. Population-wide weight loss and regain in relation to diabetes burden and cardiovascular mortality in Cuba 1980-2010: repeated cross sectional surveys and ecological comparison of secular trends. BMJ 2013346 f1515. (https://doi. org/10.1136/bmj.f1515)

15 Maple-Brown LJ, Sinha AK \& Davis EA. Type 2 diabetes in Indigenous Australian children and adolescents. Journal of Paediatrics and Child Health 201046 487-490. (https://doi.org/10.1111/j.14401754.2010.01844.x)

16 Jefferies C, Carter P, Reed PW, Cutfield W, Mouat F, Hofman PL \& Gunn AJ. The incidence, clinical features, and treatment of type 2 diabetes in children $<15 \mathrm{yr}$ in a population-based cohort from Auckland, New Zealand, 1995-2007. Pediatric Diabetes 201213 294-300. (https://doi.org/10.1111/j.1399-5448.2012.00851.x)

17 Zimmet P, Alberti KG \& Shaw J. Global and societal implications of the diabetes epidemic. Nature 2001414 782-787. (https://doi. org/10.1038/414782a)

18 Haffner SM, Stern MP, Hazuda HP, Mitchell BD \& Patterson JK. Cardiovascular risk factors in confirmed prediabetic individuals. Does the clock for coronary heart disease start ticking before the onset of clinical diabetes? JAMA 1990263 2893-2898. (https://doi. org/10.1001/jama.263.21.2893)

19 Kahn R \& Davidson MB. The reality of type 2 diabetes prevention. Diabetes Care 201437 943-949. (https://doi.org/10.2337/dc13-1954)

20 Bouton ME. Why behavior change is difficult to sustain. Preventive Medicine 201468 29-36. (https://doi.org/10.1016/j.ypmed.2014.06.010)

21 Diabetes Prevention Program Research Group, Knowler WC, Fowler SE, Hamman RF, Christophi CA, Hoffman HJ, Brenneman AT, Brown-Friday JO, Goldberg R, Venditti E et al. 10-year follow-up of diabetes incidence and weight loss in the Diabetes Prevention Program Outcomes Study. Lancet 2009374 1677-1686. (https://doi. org/10.1016/S0140-6736(09)61457-4)

22 Knowler WC, Barrett-Connor E, Fowler SE, Hamman RF, Lachin JM, Walker EA, Nathan DM \& Diabetes Prevention Program Research 
Group. Reduction in the incidence of type 2 diabetes with lifestyle intervention or metformin. New England Journal of Medicine 2002346 393-403. (https://doi.org/10.1056/NEJMoa012512)

23 Hamman RF, Wing RR, Edelstein SL, Lachin JM, Bray GA, Delahanty L, Hoskin M, Kriska AM, Mayer-Davis EJ, Pi-Sunyer X et al. Effect of weight loss with lifestyle intervention on risk of diabetes. Diabetes Care 200629 2102-2107. (https://doi.org/10.2337/dc06-0560)

24 Lindstrom J, Ilanne-Parikka P, Peltonen M, Aunola S, Eriksson JG, Hemio K, Hamalainen H, Harkonen P, Keinanen-Kiukaanniemi S, Laakso $\mathrm{M}$ et al. Sustained reduction in the incidence of type 2 diabetes by lifestyle intervention: follow-up of the Finnish Diabetes Prevention Study. Lancet 2006368 1673-1679. (https://doi. org/10.1016/S0140-6736(06)69701-8)

25 Diabetes Prevention Program Research Group. The 10-year costeffectiveness of lifestyle intervention or metformin for diabetes prevention: an intent-to-treat analysis of the DPP/DPPOS. Diabetes Care 201235 723-730. (https://doi.org/10.2337/dc11-1468)

26 Hjortebjerg R. IGFBP-4 and PAPP-A in normal physiology and disease. Growth Hormone and IGF Research 201841 7-22. (https://doi. org/10.1016/j.ghir.2018.05.002)

27 Jones JI \& Clemmons DR. Insulin-like growth factors and their binding proteins: biological actions. Endocrine Reviews 199516 3-34. (https://doi.org/10.1210/edrv-16-1-3)

28 Conover CA, Mason MA, Bale LK, Harrington SC, Nyegaard M, Oxvig C \& Overgaard MT. Transgenic overexpression of pregnancyassociated plasma protein-A in murine arterial smooth muscle accelerates atherosclerotic lesion development. American Journal of Physiology: Heart and Circulatory Physiology 2010299 H284-H291. (https://doi.org/10.1152/ajpheart.00904.2009)

29 Mazerbourg S, Overgaard MT, Oxvig C, Christiansen M, Conover CA, Laurendeau I, Vidaud M, Tosser-Klopp G, Zapf J \& Monget P. Pregnancy-associated plasma protein-A (PAPP-A) in ovine, bovine, porcine, and equine ovarian follicles: involvement in IGF binding protein-4 proteolytic degradation and mRNA expression during follicular development. Endocrinology 2001142 5243-5253. (https:// doi.org/10.1210/endo.142.12.8517)

30 Byun D, Mohan S, Yoo M, Sexton C, Baylink DJ \& Qin X. Pregnancyassociated plasma protein-A accounts for the insulin-like growth factor (IGF)-binding protein-4 (IGFBP-4) proteolytic activity in human pregnancy serum and enhances the mitogenic activity of IGF by degrading IGFBP-4 in vitro. Journal of Clinical Endocrinology and Metabolism 200186 847-854. (https://doi.org/10.1210/jcem.86.2.7223

31 Beaudeux JL, Burc L, Imbert-Bismut F, Giral P, Bernard M, Bruckert E \& Chapman MJ. Serum plasma pregnancy-associated protein A: a potential marker of echogenic carotid atherosclerotic plaques in asymptomatic hyperlipidemic subjects at high cardiovascular risk. Arteriosclerosis, Thrombosis, and Vascular Biology 200323 e7-e10. (https://doi.org/10.1161/01.atv.0000047448.76485.b8)

32 Panagiotou G, Anastasilakis AD, Kynigopoulos G, Skouvaklidou EC, Saridakis ZG, USSaAUTHSHS Study Group, Upadhyay J, Pagkalidou E, Apostolou A, Karagiozoglou-Lampoudi T et al. Physiological parameters regulating circulating levels of the IGFBP-4/ Stanniocalcin-2/PAPP-A axis. Metabolism: Clinical and Experimental 201775 16-24. (https://doi.org/10.1016/j.metabol.2017.07.003)

33 Hjortebjerg R, Laugesen E, Hoyem P, Oxvig C, Stausbol-Gron B, Knudsen ST, Kim WY, Poulsen PL, Hansen TK, Bjerre M et al. The IGF system in patients with type 2 diabetes: associations with markers of cardiovascular target organ damage. European Journal of Endocrinology 2017176 521-531. (https://doi.org/10.1530/EJE-16-0940)
34 Pellitero S, Reverter JL, Pizarro E, Pastor MC, Granada ML, Tassies D, Reverter JC, Salinas I \& Sanmarti A. Pregnancy-associated plasma protein-a levels are related to glycemic control but not to lipid profile or hemostatic parameters in type 2 diabetes. Diabetes Care 200730 3083-3085. (https://doi.org/10.2337/dc07-1092)

35 Aso Y, Okumura K, Wakabayashi S, Takebayashi K, Taki S \& Inukai T. Elevated pregnancy-associated plasma protein-a in sera from type 2 diabetic patients with hypercholesterolemia: associations with carotid atherosclerosis and toe-brachial index. Journal of Clinical Endocrinology and Metabolism 200489 5713-5717. (https://doi. org/10.1210/jc.2004-0787)

36 Mader JR, Resch ZT, McLean GR, Mikkelsen JH, Oxvig C, Marler RJ $\&$ Conover CA. Mice deficient in PAPP-A show resistance to the development of diabetic nephropathy. Journal of Endocrinology 2013 219 51-58. (https://doi.org/10.1530/JOE-13-0167)

37 Conover CA, Bale LK, Mader JR, Mason MA, Keenan KP \& Marler RJ. Longevity and age-related pathology of mice deficient in pregnancyassociated plasma protein-A. Journals of Gerontology: Series A, Biological Sciences and Medical Sciences 201065 590-599. (https://doi. org/10.1093/gerona/glq032)

38 Junnila RK, List EO, Berryman DE, Murrey JW \& Kopchick JJ. The GH/IGF-1 axis in ageing and longevity. Nature Reviews: Endocrinology 20139 366-376. (https://doi.org/10.1038/nrendo.2013.67)

39 Silahtaroglu AN, Tumer Z, Kristensen T, Sottrup-Jensen L \& Tommerup N. Assignment of the human gene for pregnancyassociated plasma protein A (PAPPA) to 9q33.1 by fluorescence in situ hybridization to mitotic and meiotic chromosomes. Cytogenetics and Cell Genetics 199362 214-216. (https://doi.org/10.1159/000133479)

40 Sagar V, Vatapalli R, Lysy B, Pamarthy S, Anker JF, Rodriguez Y, Han H, Unno K, Stadler WM, Catalona WJ et al. EPHB4 inhibition activates ER stress to promote immunogenic cell death of prostate cancer cells. Cell Death and Disease 201910 801. (https://doi. org/10.1038/s41419-019-2042-y)

41 Ruby MA, Massart J, Hunerdosse DM, Schonke M, Correia JC, Louie SM, Ruas JL, Naslund E, Nomura DK \& Zierath JR. Human carboxylesterase 2 reverses obesity-induced diacylglycerol accumulation and glucose intolerance. Cell Reports 201718 636-646. (https://doi.org/10.1016/j.celrep.2016.12.070)

42 Ware CF. The TNF superfamily-2008. Cytokine and Growth Factor Reviews 200819 183-186. (https://doi.org/10.1016/j. cytogfr.2008.05.001)

43 Kong DH, Kim YK, Kim MR, Jang JH \& Lee S. Emerging roles of vascular cell adhesion molecule-1 (VCAM-1) in immunological disorders and cancer. International Journal of Molecular Sciences 2018 19 1057. (https://doi.org/10.3390/ijms19041057)

44 BonDurant LD, Ameka M, Naber MC, Markan KR, Idiga SO, Acevedo MR, Walsh SA, Ornitz DM \& Potthoff MJ. FGF21 regulates metabolism through adipose-dependent and -independent mechanisms. Cell Metabolism 201725 935-944.e4. (https://doi. org/10.1016/j.cmet.2017.03.005)

45 Wang YS, Ye J, Cao YH, Zhang R, Liu Y, Zhang SW, Dai W \& Zhang Q. Increased serum/plasma fibroblast growth factor 21 in type 2 diabetes mellitus: a systematic review and meta-analysis. Postgraduate Medical Journal 201995 134-139. (https://doi.org/10.1136/ postgradmedj-2018-136002)

46 Davis GR, Deville T, Guillory J, Bellar D \& Nelson AG. Relationship between family history of type 2 diabetes and serum FGF21. European Journal of Clinical Investigation 201747 853-859. (https://doi. org/10.1111/eci.12835)

Received 18 March 2020

Revised version received 4 June 2020

Accepted 18 June 2020 